\title{
Sistemas de Nanopartículas Poliméricas II: Estructura, Métodos de Elaboración, Características, Propiedades, Biofuncionalización y Tecnologías de Auto-Ensamblaje Capa por Capa (Layer-by-Layer Self-Assembly)
}

\author{
Polymeric Nanoparticle Systems: Structure, Elaboration Methods, Characteristics, \\ Properties, Biofunctionalization and Self-assembly Layer by Layer Technologies
}

\author{
Madelein C. Urrejola ${ }^{1,2}$; Liliam V. Soto ${ }^{1}$; Consuelo C. Zumarán ${ }^{1,3}$; Juan Pablo Peñaloza ${ }^{1,3}$; \\ Beatriz Álvarez ${ }^{1,3}$; Ignacio Fuentevilla ${ }^{1,3}$ \& Ziyad S. Haidar ${ }^{1,3,4,5}$
}

URREJOLA, M. C.; SOTO, L. V.; ZUMARÁN, C. C.; PEÑALOZA, J. P.; ÁLVAREZ, B.; FUENTEVILLA, I. \& HAIDAR, Z. S. Sistemas de Nanopartículas Poliméricas II: estructura, métodos de elaboración, características, propiedades, biofuncionalización y tecnologías de auto-ensamblaje capa por capa (layer-by-layer self-assembly). Int. J. Morphol., 36(4):1463-1471, 2018.

RESUMEN: Los materiales poliméricos han sido ampliamente investigados para aplicaciones biomédicas, teniendo especial relevancia cuando se encuentran en forma de micro- y nano-partículas. Últimamente se ha ampliado su campo de aplicación al ser conjugados con péptidos y ácidos nucleicos, por lo tanto, el interés en el estudio de este tipo de materiales, así como también en la formulación de nanoestructuras funcionalizadas como materiales, dispositivos y vehículos de transporte de agentes terapéuticos ha aumentado. Las recientes investigaciones en nanosistemas se inspiran en fenómenos naturales que estimulan la integración de señales moleculares y la mimetización de procesos a nivel celular, de tejidos y órganos. Tecnológicamente, la capacidad de obtener nanoestructuras esféricas mediante la combinación de materiales que presenten propiedades distintas a las que ningún otro material individual posee por sí solo, es lo que hace que las nanocápsulas sean particularmente atractivas. Las potenciales ventajas de los sistemas de nanopartículas de tipo polimérico se destacan a lo largo de cada parte de este artículo de revisión. El presente artículo aborda los aspectos más relevantes sobre la estructura, composición y algunos métodos de elaboración de los sistemas nanoparticulados. Además, expone algunos de los trabajos más recientes, centrados en sistemas de nanopartículas basados en polímeros dirigidos a la administración de agentes, publicados en artículos especializados de investigación y revisiones durante los últimos años.

PALABRAS CLAVE: Nanosistemas; Nanoestructuras; Nanoesferas; Nanocápsulas; Auto-ensamblaje (L-b-L); Anfifílico; Dendrímeros; Polímeros; Ingeniería de tejidos; Quantum dots; Transporte de Droga.

\section{INTRODUCCIÓN}

El uso de nanopartículas es un campo en constante expansión y tienen un rol clave en diversas áreas como fotónica, electrónica, sensores, medicina, control de la contaminación y tecnología ambiental. Recientemente, se han encontrado variadas aplicaciones biomédicas en bioimagen, administración de fármacos y diagnóstico (Mallakpour \& Behranvand, 2016). En el área farmacéutica, se ha considerado que la utilización de partículas coloidales autoensambladas y microcápsulas es muy prometedora. Las propiedades de las nanopartículas dependen de sus características físicas, químicas o morfológicas (Bollhorst et al. 2017). Las nanopartículas coloidales se sintetizan mediante diferentes vías, sin embargo, se considera que existen cuatro principales, cada una de ellas presentan como objetivo la encapsulación de agentes farmacéuticos y su administración dirigida con fines terapéuticos (Bollhorst et al.).

\footnotetext{
${ }^{1}$ BioMAT'X, Universidad de los Andes, Las Condes, Santiago - Chile.

${ }^{2}$ Programa de Intercambio de Estudiantes en Ingeniería Biomédica, Instituto Tecnológico de Costa Rica, Cartago, Costa Rica.

${ }^{3}$ Programa de Doctorado en BioMedicina, Facultad de Medicina, Universidad de Los Andes, Las Condes, Santiago - Chile.

${ }^{4}$ Centro de Investigación e Innovación Biomédica, Universidad de Los Andes, Las Condes, Santiago - Chile.

${ }^{5}$ Facultad de Odontología, Universidad de los Andes, Las Condes, Santiago - Chile.
} 
URREJOLA, M. C.; SOTO, L. V.; ZUMARÁN, C. C.; PEÑALOZA, J. P.; ÁLVAREZ, B.; FUENTEVILLA, I. \& HAIDAR, Z. S. Sistemas de Nanopartículas Poliméricas II: estructura, métodos de elaboración, características, propiedades, biofuncionalización y tecnologías de auto-ensamblaje capa por capa (layer-by-layer self-assembly). Int. J. Morphol., 36(4):1463-1471, 2018.

Las áreas que abarca la investigación en nanopartículas de tipo coloidales son amplias y se han vuelto especialmente cotizadas, debido a que ofrecen mejores opciones terapéuticas para trastornos graves (Grossen et al., 2017) algunas de ellas se han usado incluso para tratar patologías como el cáncer. Los biomateriales compuestos de polímeros han sido ampliamente estudiados, algunos de ellos son alginato, quitosano, gelatina, ácido hialurónico, ácido poli(láctico-co-glicólico) (PLGA), polilactida (PLA), policaprolactona (PCL) y celulosa polianiónica (PAC). De los cuales los más utilizados corresponden a PLA y PCL (Kumari et al. 2010; Grossen et al.), su biodisponibilidad, encapsulamiento mejorado, control de liberación y propiedades cito y bio-compatibles mejoran el valor terapéutico de los agentes encapsulados en las nanopartículas coloidales. Por ejemplo, el PLA es ampliamente usado para diversas aplicaciones biomédicas debido a que posee propiedades como biodegradabilidad, biocompatibilidad y no toxicidad que le permiten ser utilizado en variados sistemas de transporte controlado de moléculas terapéuticas como proteínas, genes, vacunas y fármacos (Lee et al. 2016). Los estudios sobre la producción, caracterización y aplicación de nanomateriales coloidales están evolucionando rápidamente. Para nanoestructuras aplicadas en la cavidad oral, es necesario evaluar el comportamiento en distintas condiciones, contemplando factores tales como $\mathrm{pH}$, capacidad tampón de la saliva, el contacto con la mucosa y la diseminación en los tejidos dentales (Padovani et al., 2015). Cuando consideramos el uso de los nanosistemas coloidales en sistemas similares a los del cuerpo humano, podemos aprovechar al máximo sus características físicas que las hacen más especiales que otros nanomateriales. Algunas de ellas pueden estimular, responder e interactuar con células, otras pueden incluso inducir respuestas fisiológicas favorables en los tejidos dianas y con un mínimo de efectos indeseables (Whitesides, 2003). Una de las grandes metas de la aplicación de la nanotecnología en la administración de fármacos es reducir los efectos secundarios de estos, es por ello que existen algunos nanocoloides que son sensibles a estímulos externos, y son capaces de liberar los fármacos sólo en el sitio de acción deseado, disminuyendo los efectos secundarios. Es importante destacar que, gracias a la incorporación de pequeñas moléculas bioactivas en sistemas de suministro a escala nanométrica, estas pueden aumentar la biodisponibilidad del fármaco y la eficacia terapéutica, proporcionando una terapia deseable mediante la focalización (Deshmukh et al., 2013). Los sistemas layer-by-layer (LbL) o sistemas de autoensamblaje, son otro tipo herramientas nanotecnológicas prometedoras para la administración de fármacos y biosensores.

El objetivo de este artículo de revisión es (1) proporcionar breve información sobre la preparación de las nanopartículas poliméricas, algunas propiedades de su mor- fología y actividad, (2) presentar más a fondo las diversas aplicaciones terapéuticas en problemas de biomedicina, administración de fármacos y genes en ingeniería tisular, y medicina regenerativa, y finalmente (3) presentar novedades en nano-odontología.

Nanopartículas coloidales formadas por polímeros. Los polímeros son macromoléculas compuestas de un gran número de unidades repetitivas idénticas o similares. Su organización es sobre una arquitectura molecular de tipo cadena que exhibe una multiplicidad de composiciones, estructuras y propiedades. Los coloides y biocoloides se han utilizado en diversas aplicaciones desde los años setenta, aquellos basados en polisacáridos biocompatibles y biodegradables son pequeñas partículas que pueden medir entre 10 a $400 \mathrm{~nm}$ de diámetro, mientras que las micelas poliméricas pueden medir entre 10 a $50 \mathrm{~nm}$ de diámetro (Yang et al., 2006).

Según el método de preparación, existen dos tipos de nanopartículas poliméricas: nanoesferas y nanocápsulas. Las nanoesferas son sistemas matriciales esféricos, que tienen el agente activo disperso en una matriz polimérica de manera homogénea, mientras que las nanocápsulas son sistemas vesiculares que rodean el agente dentro de una cavidad rodeada por una cubierta polimérica, que controla su liberación dependiendo de su naturaleza, estas diferencias hacen que ambos tipos de nanopartículas tengan distintas propiedades en cuanto a la forma de liberar su contenido. La selección de la nanopartícula dependerá del método de elaboración, las propiedades químicas, físicas y características del fármaco a encapsular (Fig. 1).

Métodos de elaboración de nanopartículas. Existen numerosos métodos para la síntesis de nanopartículas que permiten el encapsulamiento de moléculas hidrófobas e hidrófilas. Entre los métodos de síntesis más utilizados para la elaboración de cápsulas coloidales se encuentra las emulsiones de Pickering (Bollhorst et al.) (Fig. 2). Los principales métodos de síntesis de nanopartículas se detallan a continuación:

I. Método emulsión/evaporación: Consiste en la emulsión de dos fases, una acuosa con un agente emulsionante, y una fase orgánica inmiscible en agua, con un principio activo (PA) y un polímero que formará la matriz. Para formar una fase dispersa homogénea se requiere de una fuente de energía que puede ser una sonda de ultrasonido o un sistema de agitación mecánica de velocidad variable. Formada la emulsión, comienza la evaporación del solvente orgánico, que debe ser adecuadamente volátil. El polímero precipita encapsulando principios activos hidrófobos (Lee et al.), la polimerización de una emulsión permite crear nanopartículas de tamaño y morfología controlada, para la ejecución y síntesis, en emulsiones o en soluciones que forman micelas 


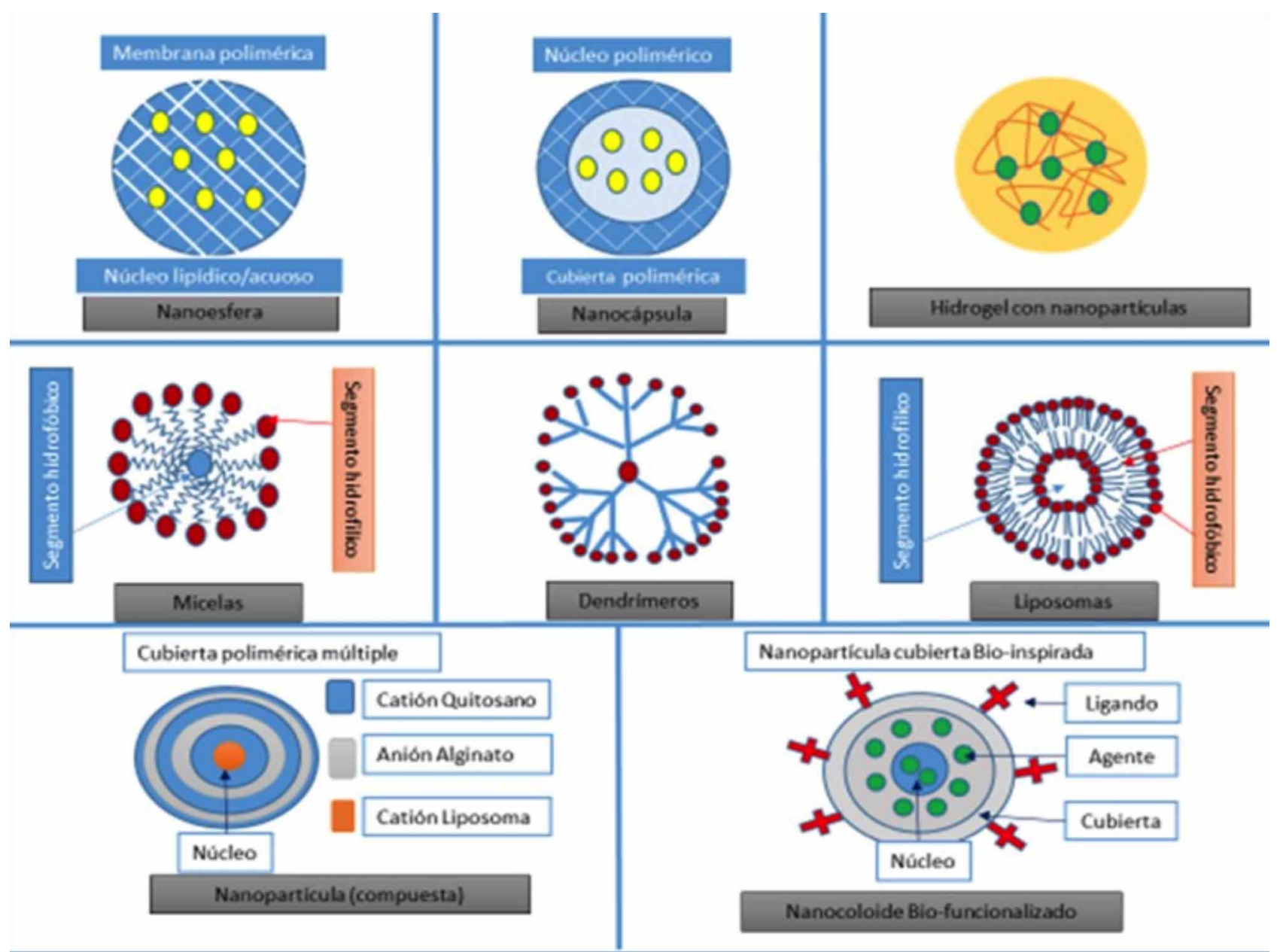

Fig. 1. Representación esquemática de nanoestructuras y sus componentes. Fila superior y fila media: Representación esquemática de tipos de nanopartículas esféricas. Fila inferior: Representación esquemática de una nanopartícula compuesta por multicapa: Layer-bylayer (L-B-L) Autoensamblada y un Nanocoloide biofuncionalizado, muy utilizado para la administración dirigida de fármacos, proteínas y genes.

(mezclando el reactante acuoso con un surfactante adecuado) seguido de oxidación, polimerización y cubierta (SolGel) (Fig. 2A).

II. Método de doble o múltiple emulsión: Este método ha permitido encapsular moléculas hidrófilas gracias a la doble emulsión (Reis et al., 2006; Lee et al.). Aquí, una molécula hidrófila se une a un surfactante y se disuelven en agua. La emulsión está compuesta por la dispersión de la fase acuosa en una fase orgánica, que presenta un polímero disuelto. Finalmente, la emulsión es sometida a evaporación, sin embargo, este procedimiento suele ser tóxico, lo que puede afectar la estabilidad de algunos componentes.

III. Método de difusión de emulsificación: Utiliza un solvente parcialmente soluble como acetona (Fig. 2B), en el que se disuelven polímeros o compuestos bioactivos, después, se emulsionan en una fase acuosa que contiene un estabiliza- dor que evita la agregación de las gotas en la emulsión. Por último, se agrega agua a la emulsión para que se disuelva el solvente y se precipitan las nanopartículas (Reis et al.).

IV. Método de Nanoprecipitación: En este método, moléculas bioactivas y el polímero se disuelven en acetona y se añade una solución acuosa que contiene tensioactivos, la acetona es evaporada y las nanopartículas permanecen en una suspensión acuosa (Fig. 2C) (Reis et al.; Lee et al.).

Durante la última década, ha existido un aumento en la demanda de nanofibras poliméricas, ya que son candidatas prometedoras para diversas aplicaciones, incluyendo ingeniería de tejidos, ropa protectora, filtración y sensores. Es por esto que se han desarrollado nuevos métodos de síntesis como el Electrospinning, esta técnica es utilizada para la fabricación de nanofibras continuas y diversos materiales (tales como polímeros, cerámicas y metales) posibilitando 


\section{A. Método emulsión/evaporación}

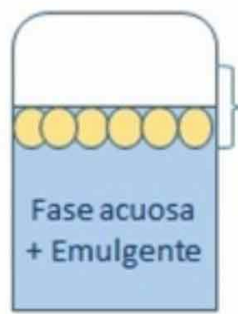

Fase orgánica:

Principio activo

+ Polímero

hidrófobo

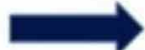

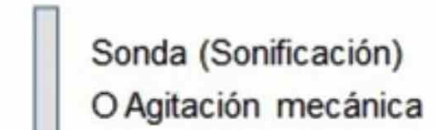

OAgitación mecánica

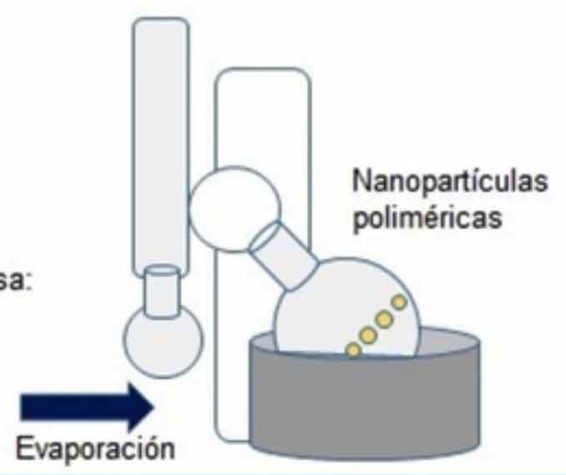

Polimero

+ PA

+ Solvente

orgánico

\section{B. Difusión de emulsificación}

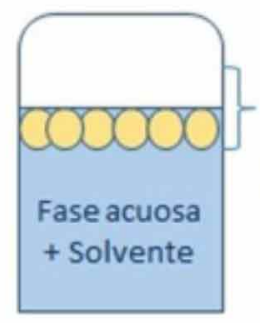

Fase orgánica:

Solvente $\left(\mathrm{C}_{3} \mathrm{H}_{6} \mathrm{O}\right)$

+ Polimero

hidrófobo

\section{Sonda (Sonificación)}

O Agitación mecánica

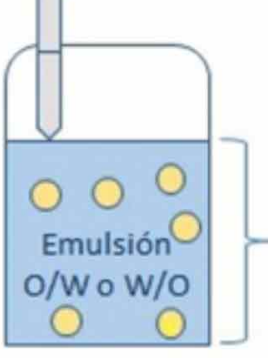

Fase Acuosa

+ Estabilizador
Fase dispersa:

Polimero

+ PA

+ Solvente

orgánico

+ estabilizador

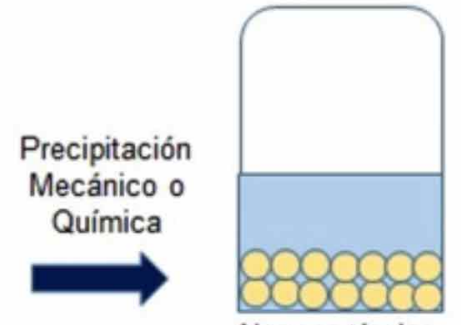

Nanopartículas poliméricas

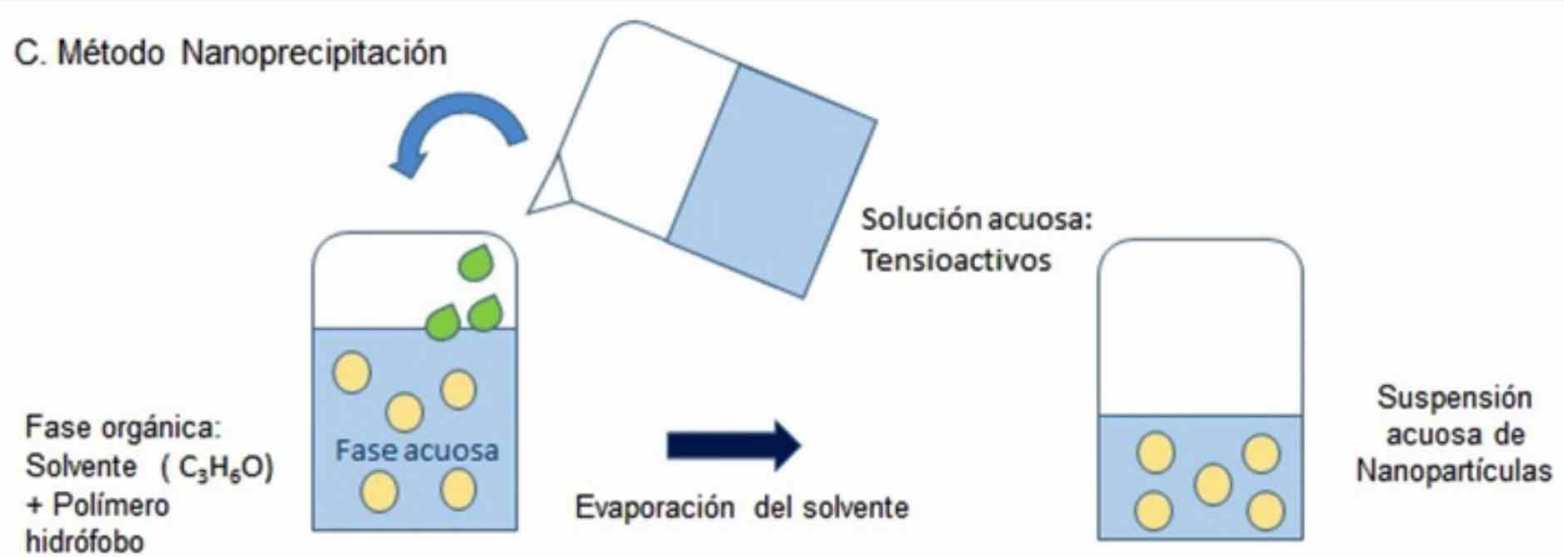

Fig. 2. Métodos de síntesis de nanopartículas poliméricas. Representación esquemática los principales métodos de síntesis. A) Emulsión/ evaporación. B) Difusión de emulsificación. C) Nanoprecipitación.

controlar el grosor de las fibras, la morfología de la superficie, la orientación y la configuración de la sección transversal. En este proceso, mediante la aplicación de una fuerza eléctrica, la solución polimérica se convierte en nanofibras sólidas (Thakkar \& Misra, 2017). Las nanofibras fabricadas por electrospinning han demostrado un gran potencial para la entrega de fármacos, debido al aumento de las tasas de disolución de la droga por el aumento de la superficie, ya que son susceptibles a modificaciones para alterar propiedades tales como el control de la velocidad de liberación, debido a la naturaleza sensible a los estímulos de algunos polímeros utilizados (Thakkar \& Misra). 
URREJOLA, M. C.; SOTO, L. V.; ZUMARÁN, C. C.; PEÑALOZA, J. P.; ÁLVAREZ, B.; FUENTEVILLA, I. \& HAIDAR, Z. S. Sistemas de Nanopartículas Poliméricas II: estructura, métodos de elaboración, características, propiedades, biofuncionalización y tecnologías de auto-ensamblaje capa por capa (layer-by-layer self-assembly). Int. J. Morphol., 36(4):1463-1471, 2018.

La selección adecuada de polímeros, mezclas poliméricas y materiales permiten a la formulación controlar numerosas propiedades asociadas con las nanofibras, logrando un éxito considerable en el campo de los productos farmacéuticos, incluyendo aplicaciones antimicrobianas, cicatrización de heridas, tratamiento del cáncer, periodoncia e ingeniería de tejidos (Thakkar \& Misra).

Características, propiedades y diseño de Nanocomplejos. Las características y propiedades de los nanomateriales puede variar producto de la diversidad en los parámetros y diseños utilizados para su síntesis. Existen variados materiales empleados como componentes principales de la matriz, pueden tener origen lipídico (Fosfolípidos, ácidos grasos), proteico (Albúmina, colágeno), polímeros naturales (Quitosano, dextrano, alginato), semisintético (derivados de celulosa) y sintéticos (poli(acrilatos), poli(acrilamidas), poli(anhídridos) y poli(ésteres)) (Gómez-Gaete, 2014).

La estructura química del polímero determinará el comportamiento en términos de encapsulación, degradación y liberación de moléculas, por ejemplo, los polímeros anfifílicos son un tipo de polímero que se autoensambla en una estructura con un núcleo cubierto, el núcleo hidrófilo sirve como depósito de genes, enzimas y una diversidad de medicamentos con diferentes características (Mishra et al., 2010), combinando el diseño con múltiples funcionalidades manteniendo la escala nanoscópica (Schärt et al. 2010). Debido al amplio abanico de posibles combinaciones de materiales, las nanopartículas con cubiertas han sido clasificadas en base al material con que se fabrica el núcleo y la cubierta/envoltura (Fig. 1).

Por ejemplo, los dendrímeros son polímeros hiperramificados, con amplia aplicación en biología y medicina, por lo que es importante entender los mecanismos fundamentales de sus interacciones con los sistemas biológicos. La fuerza electrostática desempeña el papel predominante en las interacciones dendrímero-proteína, especialmente con dendrímeros cargados, se han demostrado otros tipos de interacciones como el enlace $\mathrm{H}$, las fuerzas de van der Waals e incluso las interacciones hidrofóbicas. La unión de dendrímeros a una proteína puede cambiar su estructura secundaria, conformación, movilidad intramolecular y actividad funcional (Shcharbin et al., 2017).

Hoy en día se pueden preparar materiales complejos para imitar la arquitectura supramolecular de estructuras naturales, con capacidad añadida sofisticada y/o multifuncional. Para simplificar, según el propósito y la composición del material, las nanopartículas de ingeniería toxicológica se clasifican en: (A) Nanotubos de carbono mono capa o multicapa bidimensionales: Son largos tubos de carbono, que pueden actuar como fibras biopersistentes. Los monocapa varian con longitudes mayores a $100 \mathrm{~nm}$ y multicapa entre 2 a $50 \mathrm{~nm}$. (B) Fullerenos C60, compuesto por 60 átomos de carbono, funciona como jaulas esféricas orgánicas solubles en agua. (C) Dendrímeros: debido a su naturaleza específica son bastante adecuados para la administración de fármacos aunque su pequeño tamaño, hasta 10 $\mathrm{nm}$, limita la incorporación de moléculas en su interior. $\mathrm{Su}$ naturaleza dendrítica permite cargar moléculas en su superficie externa y pueden ser funcionalizados en su exterior con anticuerpos. Entre sus usos se han descrito su capacidad de matar células bacterianas, se han utilizado como agentes de transferencia de genes y de transporte de membrana. (D) Nanocristales esféricos semiconductores tridimensionales (1-10 nm QDs): Son un grupo heterogéneo y su toxicidad depende tanto de sus propiedades físicoquímicas como del ambiente. Los puntos cuánticos o Quantum dots, varían entre 2,5 a $100 \mathrm{~nm}$ de tamaño. Sustancias de recubrimiento bioactivo permiten permiten su uso para llegar a células y/o organelos celulares de manera específica y su toxicidad se debe a la inducción de especies reactivas de oxígeno. (E) Nanopartículas de oro/ Nanocápsulas: son ampliamente utilizadas y se pueden sintetizar en diferentes formas (varillas, puntos). Están disponibles comercialmente en diferentes tamaños y pueden detectarse a bajas concentraciones. Han sido usadas de manera compuesta con cobre y plata y se utilizan como agente de contraste en Resonancia Magnética Nuclear. (F) Nanopartículas de sílice: Tienen efecto citotóxico, pero este es dosis-dependiente (De Jong \& Borm, 2008).

Las aplicaciones y composición de estos nanocomplejos son variadas, por ejemplo, Posadowska et al. (2015) proponen nanopartículas poliméricas de PLGA como sistema de administración de gentamicina aplicado localmente en la terapia con osteomielitis. Los estudios de liberación de fármacos mostraron una liberación prolongada de gentamicina sostenida durante 35 días. Las pruebas realizadas con los patógenos causantes de osteomielitis: Staphylococcus aureus y Staphylococcus epidermidis, mostraron actividad antibacteriana de las nanopartículas cargadas GS (GS-NPs), por lo tanto, estas serían una potencial herramienta en el tratamiento de la osteomelitis (Posadowska et al.). Otros polímeros como poli(lactida) PLA y poli(caprolactona) PCL, son dos de los más utilizados, bien conocidos por su compatibilidad, biodegradabilidad, y la sostenibilidad del medio ambiente (especialmente PLA, que se puede obtener a partir de recursos renovables). Existen dieciséis formulaciones de micropartículas basadas en PLGA/PLA aprobadas por la FDA para uso clínico, pero ningún portador de nanopartículas basado en estos poliésteres ha llegado al mercado. PLGA es considerado un polímero biodegradable, biocompatible y de muy baja toxicidad, muy 
INMUNOGENOCIDAD:

- Respuesta inmune innata y adaptativa

- Activación de APCS

- Respuesta inmune antígeno-específica
DIANA CELULAR:

- Interacción con ligandos específicos de superficie celular

- Liberación intracelular específica del contenido-Ph dependiente

- Asegura una entrega directa

Fig. 3. Ejemplos de efectos informados de portadores de PLA. Partículas de PLA pueden (1) ser modificadas por diferentes entidades para alcanzar sus objetivos como antígenos, adyuvantes, fármacos, siRNAs, etc. (2) Pueden estimular la respuesta inmune específica de un antígeno, modulan la inmunosupresión y eliminan células cancerosas, mediante la regulación negativa de su proliferación. (3) Los polímeros inmunogénicos y ligandos de PPR pueden mejorar la inmunogenicidad del portador para estrategias inmunoterapéuticas. (4) pueden atrapar o ser funcionalizados con moléculas de formación de imágenes, como sondas fluorescentes, radionucleidos o agentes de contraste.

apropiado para sistemas de liberación en ambientes biológicos (Lee et al.). Por otro lado, sistemas de suministro de fármacos a nanoescala a base de copolímeros PCL/PEG representan una estrategia para implementar terapias con acumulación de fármaco mejorada en el sitio de acción y disminución de los efectos fuera de destino. Grossen et al. destacan las aplicaciones terapéuticas recientes investigadas in vitro e in vivo, utilizando sistemas avanzados tales como liberación desencadenada, terapias de múltiples componentes, entrega de fármacos no dirigidas, dirigidas y sistemas de administración génica.

\section{Nanosistemas diseñados con recubrimientos bio- funcionalizados.}

Sistemas de autoensamblado "Layer-by-Layer". Los sistemas de autoensamblado Layer by Layer (LbL) sensibles a estímulos han generado mucho interés entre los investigadores de todo el mundo debido a la simplicidad del proceso por el cual se producen y a sus numerosas aplicaciones en la administración de fármacos. Los sistemas de autoensamblaje LbL implican una adsorción alternativa simple de polielectrolitos cargados de forma opuesta sobre materiales del núcleo y son considerados herramientas prometedoras para la administración de fármacos y como biosensores.
Las películas LbL se ensamblan mediante adsorción directa repetitiva de componentes de funcionalidad opuesta. Los fármacos en forma de nanopartículas o moléculas pueden incorporarse dentro o sobre estos conjuntos multicapa, ya sea directamente o dentro de un soporte. La tecnología LbL aprovecha la interacción carga-carga entre sustrato y monocapas de polielectrolitos para crear nanoestructuras de múltiples capas unidas por fuerzas electrostáticas. La formación de estos sistemas se atribuye a interacciones electrostáticas, enlaces de hidrógeno, interacciones hidrofóbicas y fuerzas de van der Waals (Deshmukh et al.).

Las nanopartículas multifuncionales con cubierta, sintetizadas por LbL, son ampliamente utilizadas para aplicaciones biomédicas y catalíticas, por ejemplo, la generación de biosensores. Un biosensor molecular consiste en un dispositivo asociado a un proceso usado para la detección de funciones del cuerpo. Un biosensor tiene tres componentes: (1) un elemento biológico sensible, por ejemplo, una enzima, para la detección; (2) un transductor o elemento detector que transforma una señal biológica en una que puede ser fácilmente medida y cuantificada y (3) una parte electrónica para mostrar resultados de una manera fácil de usar. Las aplicaciones biosensoriales de los sistemas LbL inclu- 
URREJOLA, M. C.; SOTO, L. V.; ZUMARÁN, C. C.; PEÑALOZA, J. P.; ÁLVAREZ, B.; FUENTEVILLA, I. \& HAIDAR, Z. S. Sistemas de Nanopartículas Poliméricas II: estructura, métodos de elaboración, características, propiedades, biofuncionalización y tecnologías de auto-ensamblaje capa por capa (layer-by-layer self-assembly). Int. J. Morphol., 36(4):1463-1471, 2018.

yen principalmente biosensores enzimáticos, inmunosensores, sensores de ADN, sensores de proteínas y de ácidos nucleicos, por nombrar algunos (Deshmukh et al.).

Superficie química bioinspirada y Adaptación funcional. Entre las nanoestructuras con cubiertas más prometedoras pueden considerarse aquellas de "base polimérica", estas comprenden un núcleo polimérico y/o una envoltura polimérica, y pueden dispersarse en una matriz de cualquier clase de material, cuya propiedad vaya a ser modificada o mejorada. Cuando las nanopartículas con recubrimiento multifuncionales están biofuncionalizadas, conjugadas o revestidas pueden realizar una administración dirigida y controlada, así como, servir como biomarcadores, por ejemplo, de tumores y células cancerosas. Este diseño mejora las propiedades de estabilidad, solubilidad y citocompatibilidad de las moléculas. Los sistemas micelares, basados en polímeros que contienen un núcleo hidrófobo rodeado por polímeros hidrófilos, se suelen ensamblar a partir de un copolímero anfifílico (di-bloque o tri-bloque) y han sido ampliamente explorados como portadores de fármacos hidrofóbicos (Fig. 1). Por ejemplo, se han preparado y protegido nanocápsulas con un recubrimiento de PEG (bloque hidrófilo), estas tienen un núcleo-envoltura multifuncionales de 30-50 nm y están compuestas por micelas de copolímeros anfifilicos (copolímeros de bloque hidrófobos) de manera que permiten proteger nanopartículas bio-funcionales de $\mathrm{Au}$ o CdSe, proporcionando propiedades de detección nanoscópica, además de liberación lenta y dirigida de fármacos (Schärtl, 2010).

Alternativamente, los puntos cuánticos (QDs) fotoluminiscentes prometen muchas aplicaciones biomédicas; como una nueva clase de sondas, que muestran propiedades ópticas únicas tales como alto brillo, estabilidad y capacidad de multiplexación. Sus cubiertas presentan un material semiconductor que permite la traducción de señales medicamente traducibles para la detección biomédica in vitro y la formación de imágenes in vivo. La detección biomédica basada en QDs muestra mayor selectividad y sensibilidad que los métodos convencionales para inmunohistoquímica, inmunoensayos y ensayos enzimáticos. La aplicación de QDs en inmunohistología tinción e inmunoensayo se ha demostrado bien, sin embargo, QDs aún no han comenzado a sustituir a los tintes fluorescentes convencionales en el campo. Para traducir las propiedades únicas de QDs en aplicaciones médicas, la estabilidad coloidal y óptica de QDs en diversas condiciones biológicas debe ser mejorada desarrollando más química de superficie y protocolos de síntesis.

Además, los biosensores basados en QD permiten detecciones simples de biomarcadores múltiples. Las son- das QD, cercanas al infrarrojo muestran un gran potencial para la formación de imágenes de fluorescencia in vivo y gran capacidad para la penetración de tejido profundo, especialmente en imágenes relacionadas a la vasculatura y localización de tumores. Los QDs emisores cercanos al infrarrojo, incluyen núcleos como $\mathrm{AgSe}, \mathrm{PbSe}, \mathrm{Cd} 3 \mathrm{As} 2$ y cubiertas como $\mathrm{SiO} 2$, SdSe, Cd3P2, etc. Sin embargo, se deben determinar los parámetros de biodistribución de los QDs en el organismo con la finalidad de minimizar su toxicidad y que sean apropiados para su uso en imágenes médicas.

Existen dos enfoques para reducir la toxicidad QD: (1) usando QDs compuestos de elementos menos tóxicos como Ag2S en lugar de metales pesados; y (2) promover una eliminación rápida y segura del organismo controlando el tamaño de los QD y el carácter físico-químico de su superficie (Park et al., 2017). En un estudio reciente, Chen et al. (2015) utilizaron imágenes de la segunda ventana infrarroja cercana a los QDs (Ag2S) basadas en QDs (NIR-II 1.0-1.4 mm) para etiquetar MSC sembradas en andamios de colágeno tridimensionales (3D). Primero evaluaron la proliferación celular, luego los factores de transcripción asociados a la pluripotencia y finalmente la diferenciación osteogénica como adipogénica de las MSC cultivadas en 3D (Chen et al.).

Lógicamente, a mayor tiempo de circulación aumentará la probabilidad de que las nanopartículas y sus cargas alcancen los objetivos previstos. La modificación superficial, síntesis y elección de materiales poliméricos para formular portadores de cubierta nanométrica dependen totalmente de la naturaleza de los fármacos, los períodos de suministro, la estabilidad, la permeabilidad y los perfiles de liberación del fármaco. Por lo tanto, si se requiere una circulación sistémica sostenida, las superficies de las nanopartículas hidrofóbicas deben ser modificadas para no ser atrapadas en el hígado o bazo. La presencia de cadenas de PEG en la cubierta otorga una codiciada ventaja, ya que aumentan el tiempo de circulación sanguínea, frente a aquellas nanopartículas que carecen de esta capa. Este método de camuflaje crea una capa o envoltura protectora hidrófila alrededor de la nanopartícula, otorgándole capacidad de absorción de proteínas como opsonina, a través de fuerzas de repulsión estérica, bloqueando y retrasando el proceso de opsonización (Kumari et al.; De Jong \& Borm). Esta es la principal razón por la que las nanopartículas pequeñas (menores a $100 \mathrm{~nm}$ de diámetro, como micelas poliméricas) pueden evitar el reconocimiento de los sistemas de defensa celular (SFM) después de modificar la superficie, por lo tanto, circular por más tiempo (Kumari et al.). Estos tamaños únicos, tan pequeños de las nanopartículas, es obtenible gracias a la funcionalización de la superficie o modificación de esta, hasta alcanzar una característica deseada para una aplicación específica (Fig. 1). 
URREJOLA, M. C.; SOTO, L. V.; ZUMARÁN, C. C.; PEÑALOZA, J. P.; ÁLVAREZ, B.; FUENTEVILLA, I. \& HAIDAR, Z. S. Sistemas de Nanopartículas Poliméricas II: estructura, métodos de elaboración, características, propiedades, biofuncionalización y tecnologías de auto-ensamblaje capa por capa (layer-by-layer self-assembly). Int. J. Morphol., 36(4):1463-1471, 2018.

La modificación superficial puede realizarse recubriendo el núcleo con una capa protectora hidrófila estática, tal como derivados de PEG, PVP o Quitosano, que evitan que las macromoléculas interactúen con la nanopartícula, incluso con una baja cobertura superficial para liberar su carga en un sitio específico. Otros estudios han revelado que los PEGylated-QDs escapan al SFM enfatizando la protección de la nanoescala; una oportunidad única para su uso extensivo en animales y humanos (Kumari et al.).

Yang et al. (2017) obtuvieron resultados que demuestran que la PEGilación con PEG de mayor peso molecular, inhibe significativamente la fagocitosis de macrófagos y la interacción inespecífica con glóbulos rojos, lo que transforma a esta técnica de recubrimiento en una estrategia prometedora para mejorar el rendimiento de nanopartículas con Quitosano/siRNA, tanto in vitro como in vivo.

También se han utilizado nanopartículas de PLA para la administración de fármacos y vacunas ya que protegen a las enzimas plasmáticas, induciendo su liberación sostenida y dirigida de antígenos y adyuvantes asociados a bacterias, virus y tumores in vivo, desencadenando diferentes respuestas inmunes. El PLA es considerado como un polímero prometedor para el desarrollo de potentes sistemas de administración de vacunas contra patógenos y cáncer (Peres et al., 2017; Whitesides).

Nanosistemas con cubiertas poliméricas y aplicaciones. La nanotecnología ha acelerado considerablemente el crecimiento de la medicina regenerativa en años recientes, revolucionando el diseño de injertos y andamios dando lugar a nuevos sistemas que presentan propiedades de regeneración celular y tisular significativamente mejores. Dado que la interacción entre célula y matriz celular tiene lugar a escala nanométrica, la aplicación de la nanotecnología da una ventaja en la modificación de la función celular y/o función de la matriz de manera tal de imitar el tejido u órgano nativo. Recientes avances y tendencias de la medicina regenerativa incluyen aplicaciones en órganos tales como hueso, cartílago, nervio, piel, dientes, miocardio, hígado y bulbo ocular.

Los traumatismos y las enfermedades frecuentemente producen fracturas o defectos óseos de tamaño crítico y su manejo a veces requiere de injerto óseo. El proceso de curación y regeneración ósea involucra una intrincada red de moléculas, entre las que se encuentran proteínas morfogenéticas óseas (BMPs), proteínas pertenecientes a una superfamilia de proteínas y que tienen propiedades y aplicaciones muy prometedoras por lo que han sido estudiadas intensivamente en el área de regeneración ósea. Se han identificado más de 20 tipos de BMPs, pero sólo un subconjunto de BMPs puede inducir la formación ósea de novo. Muchos grupos de investigación han demostrado que las BMP pueden inducir la diferenciación de células madre mesenquimales y células madre en células osteogénicas capaces de producir hueso (Sheikh et al., 2015).

Por otra parte, nuevas estrategias de tratamiento regenerador de la piel están dirigidos al desarrollo de andamios biológicamente sensibles capaces de suministrar múltiples agentes y células bioactivas a los tejidos diana. Norouzi et al. (2015) encapsularon EGF en nanofibras de PLGA y gelatina, a través de electrospinning. Los resultados obtenidos muestran una bioactividad deseable y hemostasia de los andamios, con capacidad de encapsulación y liberación controlada de la proteína, por lo tanto, serviría como andamios para el vendaje de heridas e ingeniería de tejidos (Norouzi et al.).

Kundu et al. (2015) sintetizaron andamios de PCL/ alginato impresos en celdas 3D, que encapsulan condrocitos y TGFb. La impresión celular sobre los hidrogeles de alginato, no presentaba efectos en la viabilidad de los condorcitos, además, facilitó la construcción del andamio. Las evaluaciones in vitro, sugieren formación y síntesis de ECM y GAG de los condorcitos, y hasta cuatro semanas después, no existían efectos adversos. De esta manera, desarrollaron con éxito un andamio para la regeneración de cartílago (Kundu et al.).

\section{CONCLUSIONES}

La evolución de los métodos de preparación de nanopartículas se ha caracterizado por la necesidad de sintetizar compuestos no tóxicos y viables, simplificar los procedimientos y mejorar de manera óptima el rendimiento y la eficiencia en el encapsulamiento. Existen variadas técnicas que son simples y seguras para encapsular fármacos, tales como nanocápsulas, nanoesferas, nanofibras, etc; que debido a las características fisicoquímicas de sus principios activos, así como su carga superficial, presentan las propiedades deseadas.

Una superficie bio-funzionalizada permite una liberción más controlada y dirigida. Los usos de nanopartículas son amplios y su desarrollo muy prometedor, sin embargo, a pesar de que son materiales altamente competentes, también presentan limitaciones para generar un tamaño uniforme y una alta eficacia de encapsulamiento. A pesar de los avances que se han realizado, las aplicaciones de este tipo de biomateriales es muy amplia y quedan muchas por desarollar. 
URREJOLA, M. C.; SOTO, L. V.; ZUMARÁN, C. C.; PEÑAlOZA, J. P.; ÁlVAREZ, B.; FUENTEVILLA, I. \& HAIDAR, Z. S. Sistemas de Nanopartículas Poliméricas II: estructura, métodos de elaboración, características, propiedades, biofuncionalización y tecnologías de auto-ensamblaje capa por capa (layer-by-layer self-assembly). Int. J. Morphol., 36(4):1463-1471, 2018.

AGRADECIMIENTOS. Este trabajo fue apoyado por las subvenciones de funcionamiento otorgadas al BioMAT'X (Laboratorio de Biomateriales, Farmacéuticos y Bioingeniería de Tejidos Cráneo Máxilo-Facial), miembro del CIIB (Centro de Investigación e Innovación Biomédica), a través de la Facultad de Odontología y Fondo de Ayuda a la Investigación (FAI No. INV-IN-2015-101 2015-2019), Dirección de Investigación, Universidad de los Andes, Santiago de Chile y CONICYTFONDEF (Grant ID \# 16I10366).

URREJOLA, M. C.; SOTO, L. V.; ZUMARÁN, C. C.; PEÑALOZA, J. P.; ÁLVAREZ, B.; FUENTEVILLA, I. \& HAIDAR, Z. S. Polymeric Nanoparticle Systems II: structure, elaboration methods, characteristics, properties, biofunctionalization and self-assembly layer by layer technologies. Int. J. Morphol., 36(4):1463-1471, 2018.

SUMMARY: Polymeric materials have been extensively investigated for biomedical applications including micro- and nanoparticles. Modern advances have broadened horizons for application with peptides and nucleic acids. Therefore, interests increased in the formulation of materials, devices and vehicles for transporting therapeutic agents in functionalized nanostructures. Recent nano-systems are inspired by natural phenomena that stimulate the integration of molecular signals and the mimicking of natural cellular processes, at tissue and organ levels. Technologically, the ability to obtain spherical nanostructures, which combine different properties, that no other single material possesses on its own, makes nanocapsules particularly attractive. Potential advantages over polymer nanoparticulate systems are highlighted throughout each part of this review article. Here, we address the most relevant aspects of structure, composition and methods of formulation of nanoparticulate systems. In addition, we outline some of the more recent works focusing on nanosized preparations, based on agent-directed polymers, found in specialized research articles that have emerged in the recent years.

KEY WORDS: Nanostructures; Nanospheres; Nanocapsules; Dendrimers; Quantum dots.

\section{REFERENCIAS BIBLIOGRÁFICAS}

Bollhorst, T.; Rezwan, K. \& Maas, M. Colloidal capsules: nano- and microcapsules with colloidal particle shells. Chem. Soc. Rev., 46(8): 2091126, 2017.

Chen, G.; Tian, F.; Li, C.; Zhang, Y.; Weng, Z.; Zhang, Y.; Peng, R. \& Wang, Q. In vivo real-time visualization of mesenchymal stem cells tropism for cutaneous regeneration using NIR-II fluorescence imaging. Biomaterials, 53:265-73, 2015.

De Jong, W. H. \& Borm, P. J. A. Drug delivery and nanoparticles: Applications and hazards. Int. J. Nanomedicine, 3(2):133-49, 2008.

Deshmukh, P. K.; Ramani, K. P.; Singh, S. S.; Tekade, A. R.; Chatap, V. K.; Patil, G. B. \& Bari, S. B. Stimuli-sensitive layer-by-layer (LbL) selfassembly systems: targeting and biosensory applications. J. Control Release, 166(3):294-306, 2013

Gómez-Gaete, C. Nanopartículas poliméricas: tecnología y aplicaciones farmacéuticas. Rev. Farmacol. Chile, 7(2):7-16, 2014.

Grossen, P.; Witzigmann, D.; Sieber, S. \& Huwyler, J. PEG-PCL-based nanomedicines: A biodegradable drug delivery system and its application. J. Control Release, 260:46-60, 2017.
Kumari, A.; Yadav, S. K. \& Yadav, S. C. Biodegradable polymeric nanoparticles based drug delivery systems. Colloids Surf. B Biointerfaces, 75(1):1-18, 2010.

Kundu, J.; Shim, J. H.; Jang, J.; Kim, S. W. \& Cho, D. W. An additive manufacturing-based PCL-alginate-chondrocyte bioprinted scaffold for cartilage tissue engineering. J. Tissue Eng. Regen. Med., 9(11):1286-97, 2015

Lee, B. K.; Yun, Y. \& Park, K. PLA micro- and nano-particles. Adv. Drug Deliv. Rev., 107:176-91, 2016.

Mallakpour, S. \& Behranvand, V. Polymeric nanoparticles: Recent development in synthesis and application. eXPRESS Polym. Lett., 10(11):895-913, 2016.

Mishra, B.; Patel, B. B. \& Tiwari, S. Colloidal nanocarriers: a review on formulation technology, types and applications toward targeted drug delivery. Nanomedicine, 6(1):9-24, 2010.

Norouzi, M.; Shabani, I.; Ahvaz, H. H. \& Soleimani, M. PLGA/gelatin hybrid nanofibrous scaffolds encapsulating EGF for skin regeneration. J. Biomed. Mater. Res. A, 103(7):2225-35, 2015.

Padovani, G. C.; Feitosa, V. P.; Sauro, S.; Tay, F. R.; Durán, G.; Paula, A. J. \& Durán, N. Advances in dental materials through nanotechnology: facts, perspectives and toxicological aspects. Trends Biotechnol., 33(11):621-36, 2015.

Park, Y.; Jeong, S. \& Kim, S. Medically translatable quantum dots for biosensing and imaging. J. Photochem. Photobiol. C Photochem. Rev., 30:51-70, 2017.

Peres, C.; Matos, A. I.; Conniot, J.; Sainz, V.; Zupancic, E.; Silva, J. M.; Graça, L.; Sá Gaspar, R.; Préat, V. \& Florindo, H. F. Poly(lactic acid)-based particulate systems are promising tools for immune modulation. Acta Biomater., 48:4157, 2017.

Posadowska, U.; Brzychczy-Woch, M. \& Pamua, E. Gentamicin loaded PLGA nanoparticles as local drug delivery system for the osteomyelitis treatment. Acta Bioeng. Biomech., 17(3):41-8, 2015.

Reis, C. P.; Neufeld, R. J.; Ribeiro, A. J. \& Veiga, F. Nanoencapsulation I. Methods for preparation of drug-loaded polymeric nanoparticles. Nanomedicine, 2(1):8$21,2006$.

Schärtl, W. Current directions in core-shell nanoparticle design. Nanoscale, 2(6):829-43, 2010

Shcharbin, D.; Shcharbina, N.; Dzmitruk, V.; Pedziwiatr-Werbicka, E.; Ionov, M.; Mignani, S.; de la Mata, F. J.; Gómez, R.; Muñoz-Fernández, M. A.; Majoral, J. P. \& Bryszewska, M. Dendrimer-protein interactions versus dendrimer-based nanomedicine. Colloids Surf. B Biointerfaces, 152:414-22, 2017.

Sheikh, Z.; Javaid, M. A.; Hamdan, N. \& Hashmi, R. Bone regeneration using bone morphogenetic proteins and various biomaterial carriers. Materials (Basel), 8(4):1778-816, 2015.

Thakkar, S. \& Misra, M. Electrospun polymeric nanofibers: New horizons in drug delivery. Eur. J. Pharm. Sci., 107:148-67, 2017.

Whitesides, G. The 'right' size in nanobiotechnology. Nat. Biotechnol., 21(10):1161-5, 2003

Yang, C.; Gao, S.; Dagnæs-Hansen, F.; Jakobsen, M.\& Kjems, J. Impact of PEG chain length on the physical properties and bioactivity of PEGylated Chitosan/ siRNA nanoparticles in vitro and in vivo. ACS Appl. Mater. Interfaces, 9(14):12203-16, 2017

Yang, Y. Y.; Wang, Y.; Powell, R. \& Chan, P. Polymeric core-shell nanoparticles for therapeutics. Clin. Exp. Pharmacol. Physiol., 33(5-6):557-62, 2006.

Dirección para correspondencia:

Ziyad S. Haidar. DDS.

Cert Implantol, MSc OMFS, FRCS(C), MBA, PhD.

Professor and Scientific Director, Faculty of Dentistry

Universidad de Los Andes

Las Condes

Santiago - CHILE

E-mail: zhaidar@uandes.cl

Recibido: $15-05-2018$

Aceptado: 28-09-2018 